\title{
K3 surfaces over number fields with geometric Picard number one
}

\author{
Jordan S. Ellenberg * \\ Princeton University \\ ellenber@math . princeton. edu
}

22 Dec 2002

A long-standing question in the theory of rational points of algebraic surfaces is whether a K3 surface $X$ over a number field $K$ acquires a Zariski-dense set of $L$-rational points over some finite extension $L / K$. In this case, we say $X$ has potential density of rational points. In case $X_{\mathbb{C}}$ has Picard rank greater than 1, Bogomolov and Tschinkel [2] have shown in many cases that $X$ has potential density of rational points, using the existence of elliptic fibrations on $X$ or large automorphism groups of $X$. By contrast, we do not know a single example of a K3 surface $X / K$ with geometric Picard number 1 which can be shown to have potential density of rational points; nor is there an example which we can show not to have potential density of rational points. In fact, the situation is even worse; the moduli space of polarized K3 surfaces of a given degree contains a countable union of subvarieties, each parametrizing a family of K3 surfaces with geometric Picard number greater than 1 . Since $\overline{\mathbb{Q}}$ is countable, it is not a priori obvious that these subvarieties don't cover the $\overline{\mathbb{Q}}$-points of the moduli space. In other words, it is a non-trivial fact that there exists a K3 surface over any number field with geometric Picard number 1 !

In this note, we correct this slightly embarrassing situation by proving the following theorem:

Theorem 1. Let $d$ be an even positive integer. Then there exists a number field $K$ and a polarized $K 3$ surface $X / K$, of degree $d$, such that $\operatorname{rank} \operatorname{Pic}\left(X_{\mathbb{C}}\right)=1$.

The main idea is to use an argument of Serre on $\ell$-adic groups to reduce the problem to proving the existence of K3 surfaces whose associated mod-n Galois representations have large image for some finite $n$; we then use Hilbert's irreducibility theorem and global Torelli for K3's to complete the proof.

Acknowledgment: This note is the result of a conversation between the author, Brendan Hassett, and A.J. de Jong, which took place at the American Institute of Mathematics during the workshop, "Rational and integral points on higher-dimensional varieties." It should also be pointed out that the main idea, in case $d=4$, is implicit in the final remark of [3].

We begin by recalling some notations and basic facts regarding K3 surfaces. An element $x$ of an abelian group $L$ is called primitive if it is not contained in $k L$ for any integer $k>1$. Let $X$ be a $\mathrm{K} 3$ surface over a number field $K$, and write $\bar{X}$ for $X \times_{K} \bar{K}$. The group $H^{2}\left(X_{\mathbb{C}}, \mathbb{Z}\right)$ is isomorphic to $\mathbb{Z}^{22}$; the cup product on $H^{2}\left(X_{\mathbb{C}}, \mathbb{Z}\right)$ is a quadratic form with signature $(3,19)$, which we denote

\footnotetext{
*Partially supported by NSA Young Investigator Grant MDA905-02-1-0097.
} 
$\langle$,$\rangle . A polarized K 3$ surface is a pair $(X, \mathcal{L})$, where $X / K$ is a $\mathrm{K} 3$ surface and $\mathcal{L}$ is an ample line bundle on $X$. If $X$ is a polarized $\mathrm{K} 3$, we let $x$ be the class of $\mathcal{L}$ in $H^{2}\left(X_{\mathbb{C}}, \mathbb{Z}\right)$; then the positive even integer $\langle x, x\rangle$ is called the degree of $X$. We denote by $L_{X}$ the orthogonal complement of $x$ in $H^{2}\left(X_{\mathbb{C}}, \mathbb{Z}\right)$. Denote by $\Gamma$ the group of isometries of $H^{2}\left(X_{\mathbb{C}}, \mathbb{Z}\right)$ which fix $x$ and which lie in the identity component of $\operatorname{Aut}\left(H^{2}\left(X_{\mathbb{C}}, \mathbb{R}\right)\right)$. So $\Gamma$ is an arithmetic subgroup of $S O(2,19)(\mathbb{Q})$.

For each prime $\ell$ we denote by $G_{\ell}$ the group of linear transformations $\alpha$ of $L_{X} \otimes_{\mathbb{Z}} \mathbb{Z}_{\ell}$ such that there exists $\chi(\alpha) \in \mathbb{Z}_{\ell}^{*}$ satisfying

$$
\langle\alpha x, \alpha x\rangle=\chi(\alpha)\langle x, x\rangle
$$

for all $x \in L_{X} \otimes_{\mathbb{Z}} \mathbb{Z}_{\ell}$. There is a natural inclusion

$$
\iota: \Gamma \rightarrow G_{\ell}
$$

and we denote by $H_{\ell}$ the closure, in the $\ell$-adic topology, of $\iota(\Gamma)$.

When a polarized K3 surface $X$ is defined over a number field $K$, the inclusion

$$
L_{X} \otimes_{\mathbb{Z}} \mathbb{Z}_{\ell} \subset H^{2}\left(\bar{X}, \mathbb{Z}_{\ell}\right)
$$

induces a $\operatorname{Gal}(\bar{K} / K)$-module structure on $L_{X} \otimes_{\mathbb{Z}} \mathbb{Z}_{\ell}$; we denote by

$$
\rho_{X}: \operatorname{Gal}(\bar{K} / K) \rightarrow G_{\ell}
$$

the resulting $\ell$-adic Galois representation.

We begin by showing that the desired statement about Pic $X_{\mathbb{C}}$ follows if the image of $\rho_{X}$ is large enough.

Lemma 2. Let $\ell$ be a prime. Suppose $\rho_{X}(\operatorname{Gal}(\bar{K} / K))$ contains a finite-index subgroup of $H_{\ell}$. Then $\operatorname{rank} \operatorname{Pic} X_{\mathbb{C}}=1$.

Proof. Suppose rank $\operatorname{Pic}\left(X_{\mathbb{C}}\right)$ is greater than 1 ; that is, there is divisor on $X_{\mathbb{C}}$ whose class is linearly independent from the class of the polarization. This divisor can be defined over some finite extension $L / K$. It follows that $\rho_{X}(\operatorname{Gal}(\bar{K} / L))$ is contained in the stabilizer of a line in $L_{X} \otimes_{\mathbb{Z}} \mathbb{Z}_{\ell}$. But this stabilizer does not contain a finite-index subgroup of $H_{\ell}$.

We also need a general lemma on linear $\ell$-adic groups.

Lemma 3. Let $H$ be a closed subgroup of $\mathrm{GL}_{m}\left(\mathbb{Z}_{\ell}\right)$. Let $\Gamma_{H}\left(\ell^{n}\right)$ be the kernel of projection from $H$ to $\mathrm{GL}_{m}\left(\mathbb{Z} / \ell^{n} \mathbb{Z}\right)$. Then there exists an integer $N$ such that no proper closed subgroup of $H$ projects surjectively onto $H / \Gamma_{H}\left(\ell^{N}\right)$.

Proof. Since $H$ is a closed subgroup of $\mathrm{GL}_{m}\left(\mathbb{Z}_{\ell}\right)$, it is an analytic subgroup. In particular, there is a subspace $L \subset M_{m}\left(\mathbb{Q}_{\ell}\right)$ and a positive integer $N$ such that, for all $n \geq N$, the group $\Gamma_{H}\left(\ell^{n}\right)$ is precisely the set of matrices $\exp (\lambda)$, where $\lambda$ ranges over $\ell^{n} M_{m}\left(\mathbb{Z}_{\ell}\right) \cap L$. Thus, every element of $\Gamma_{H}\left(\ell^{n}\right)$ can be written as $\exp (\ell \lambda)$ for some $\lambda \in L$; in particular, for every $u \in \Gamma_{H}\left(\ell^{n}\right)$ there exists $v \in \Gamma_{H}\left(\ell^{n-1}\right)$ with $v^{\ell}=u$. (See [4] for basic facts used here about $\ell$-adic Lie groups.) We also require $N \geq 2$.

We now proceed as in [6, IV.3.4, Lemma 3], which proves the lemma in the case $H=\mathrm{SL}_{2}$. Suppose $H_{0}$ is a closed subgroup projecting surjectively onto $H / \Gamma_{H}\left(\ell^{N}\right)$. It suffices to prove that $H_{0}$ projects surjectively onto $H / \Gamma_{H}\left(\ell^{n}\right)$ for all $n>N$. We proceed by induction and assume $H_{0}$ projects surjectively onto $H / \Gamma_{H}\left(\ell^{n-1}\right)$. We therefore need only show that, for all $x \in \Gamma_{H}\left(\ell^{n-1}\right)$, there exists $h \in H_{0}$ with $h^{-1} x \in \Gamma_{H}\left(\ell^{n}\right)$. Since $n-1 \geq N$, there exists $y \in \Gamma_{H}\left(\ell^{n-2}\right)$ such that 
$y^{\ell}=x$. We may write $y=1+\ell^{n-2} Y+\ell^{n-1} M_{1}$ for matrices $Y, M_{1} \in M_{m}\left(\mathbb{Z}_{\ell}\right)$. By hypothesis, there exists $h^{\prime} \in H_{0}$ such that $\left(h^{\prime}\right)^{-1} y \in \Gamma_{H}\left(\ell^{n-1}\right)$. Then

$$
h^{\prime}=1+\ell^{n-2} Y+\ell^{n-1} M_{2} .
$$

for some $M_{2} \in \mathrm{GL}_{m}\left(\mathbb{Z}_{\ell}\right)$. So take

$$
h=\left(h^{\prime}\right)^{\ell}=1+\ell^{n-1} Y+\ell^{n} M_{2}+(1 / 2)(\ell)(\ell-1) \ell^{2 n-3} Y^{2}+\ldots
$$

which is congruent to $x \bmod \ell^{n}$, since $n>N \geq 2$.

The purpose of Lemma 3 is to reduce the problem of showing that an $\ell$-adic representation has large image to the corresponding problem for a $\bmod \ell^{N}$ representation. Below we show how to use Hilbert irreducibility to produce K3 surfaces $X$ such that $\rho_{X}$ has large image $\bmod \ell^{N}$, where $N>0$ is an integer to be specified at the end.

Write $L_{d}$ for the rank-21 lattice $\langle-d\rangle \oplus H \oplus H \oplus E_{8} \oplus E_{8}$. Then $L_{X}$ is isomorphic to $L_{d}$ for any polarized K3 of degree $d$.

By a level $m$ structure on a polarized K3 we mean a choice of isometry

$$
\phi: L_{X} / m L_{X} \cong L_{d} / m L_{d} .
$$

We denote by $\Gamma(m)$ the kernel of the map $\Gamma \rightarrow \mathrm{GL}\left(L_{d} / m L_{d}\right)$. Choose a $p$ large enough so that $\Gamma(p)$ is a torsion-free group. (It suffices to choose $p$ larger than the order of any finite-order element of $G L\left(L_{d}\right)$.) If $(X, \phi)$ is a polarized K3 with level $p$ structure, any automorphism $\alpha: X \rightarrow X$ preserving the polarization and $\phi$ must have finite order (because it preserves the polarization) and thus must act trivially on $L_{X}$ (by the hypothesis on $p$ ). But then $\alpha$ is trivial by the Torelli theorem for K3's [5].

Let $\mathcal{M} / \mathbb{Q}$ be the moduli space of pairs $\left(X, \phi_{p}\right)$, where $X$ is a polarized K3 surface of degree $d$ and $\phi_{p}$ is a level $p$ structure, with $p \neq \ell$. We can construct this moduli space by GIT, as in the final remark of [1]. The fact that $\left(X, \phi_{p}\right)$ admits no nontrivial automorphisms implies that $\tilde{\mathcal{M}}$ is a fine moduli space. Now let $\tilde{\mathcal{M}}\left(\ell^{N}\right)$ be the space of pairs $\left(X, \phi_{p}, \phi_{\ell^{N}}\right)$, where $\phi_{\ell^{N}}$ is a level $\ell^{N}$ structure on $X$. Note that $\tilde{\mathcal{M}}$ and $\mathcal{M}\left(\ell^{N}\right)$ are not a priori connected.

Using again the Torelli theorem for K3 surfaces, we know that the analytic moduli space of polarized K3 surfaces of degree $d$ is a quotient $\Gamma \backslash \Omega$, where $\Omega$ is a certain connected 19-dimensional domain of periods. (See $[1, \S 3]$, noting that our $\Gamma$ is an index-2 subgroup of Beauville's $\Gamma_{q}$.) It follows that $\Gamma(p) \backslash \Omega$ is a connected component of the analytification $\tilde{\mathcal{M}}^{\text {an }}$ of $\tilde{\mathcal{M}}$, and $\Gamma\left(p \ell^{N}\right) \backslash \Omega$ is a connected component of $\tilde{\mathcal{M}}\left(\ell^{N}\right)^{a n}$. Denote by $\mathcal{M}$ and $\mathcal{M}\left(\ell^{N}\right)$ the connected components of $\tilde{\mathcal{M}}$ and $\tilde{\mathcal{M}}\left(\ell^{N}\right)$ corresponding to the quotients above; then, for some number field $K$, the map $\pi: \mathcal{M}\left(\ell^{N}\right) \rightarrow \mathcal{M}$ is a Galois cover of varieties over $K$ with Galois group $\Gamma(p) / \Gamma\left(p \ell^{N}\right)$. Denote this finite group by $\bar{\Gamma}$.

Now let $p: \mathcal{M} \rightarrow \mathbb{P}^{19}$ be a generically finite map of degree $n$. Then the composition $p \circ \pi$ expresses the function field $K\left(\mathcal{M}\left(\ell^{N}\right)\right)$ as a finite extension of $K\left(\mathbb{P}^{19}\right)$. Let $U$ be a Galois cover of $\mathbb{P}^{19}$ whose function field is the Galois closure of $K\left(\mathcal{M}\left(\ell^{N}\right)\right) / K\left(\mathbb{P}^{19}\right)$. Then the Galois group $G$ of $K(U) / K\left(\mathbb{P}^{19}\right)$ is naturally contained in the wreath product $W$ of $\bar{\Gamma}$ with $S_{n}$. The group $W$ fits in an exact sequence

$$
1 \rightarrow \bar{\Gamma}^{n} \rightarrow W \rightarrow S_{n} \rightarrow 1
$$

and the intersection of $G$ with a Cartesian factor of $\bar{\Gamma}^{n}$ is the full group $\bar{\Gamma}$, since $\bar{\Gamma}$ is the Galois group of the cover $\pi$. 
Now, by the Hilbert irreducibility theorem, there is a Zariski-dense subset of $\mathbb{P}^{19}(K)$ consisting of points $x$ such that the Galois group of $(p \circ \pi)^{-1}(x)$ over $x$ is the full group $G$. Let $x$ be such a point, and let $y$ be a $\overline{\mathbb{Q}}$-point of $\mathcal{M}$ lying over $x$. Then $y \in \mathcal{M}(L)$ for some number field $L$, and the Galois group of $\pi^{-1}(y)$ over $y$ is the full group $\bar{\Gamma}$. If $X / L$ is the $\mathrm{K} 3$ surface corresponding to the point $y$, the map

$$
\operatorname{Gal}(\overline{\mathbb{Q}} / L) \rightarrow G L_{2}\left(L_{X} \otimes_{\mathbb{Z}}\left(\mathbb{Z} / \ell^{N} \mathbb{Z}\right)\right)
$$

given by the Galois action on $H_{e t}^{2}\left(X, \mathbb{Z} / \ell^{N} \mathbb{Z}\right)$ has image $\bar{\Gamma}$. Now apply Lemma 3 , taking $H$ to be the closure in the $\ell$-adic topology of the image of $\Gamma(p)$ in $G L_{2}\left(L_{X} \otimes_{\mathbb{Z}} \mathbb{Z}_{\ell}\right)$. We conclude that, having chosen $N$ large enough, we can find a degree $d$ polarized K3 surface $X$ over a number field $L$ such that the image of $\rho_{X}$ contains $H$, which is a finite-index subgroup of $H_{\ell}$. Now $X$ has geometric Picard number 1 by Lemma 2 .

Remark 4. Lemmas 2 and 3, in principle, should allow one to write down a K3 of any desired degree which has geometric Picard number 1. One would first compute suitable values of $\ell$ and $N$, as Lemma 3 guarantees we can. It remains to write down a K3 surface $X$ such that the representation of Galois on $H_{e t}^{2}\left(X, \mathbb{Z} / \ell^{N} Z\right)$ is as large as possible. In case $d=4$, this computation is precisely the one suggested in the final remark of [3]. In order to make this computation more tractable, it might be a good idea to restrict to a family of quartic surfaces whose monodromy group $\Gamma_{0}$ is smaller than $\Gamma$, but which still doesn't have any stabilizers of points in $L_{X}$ as finite-index subgroups.

\section{References}

[1] Beauville, A. Application aux espaces de modules. Geometry of K3 surfaces: moduli and periods (Palaiseau, 1981/1982). Astérisque No. 126 (1985), 141-152.

[2] Bogomolov, F. and Tschinkel, Y. Density of rational points on elliptic K3 surfaces. Asian J. Math. 4 (2000), no. 2, 351-368.

[3] T. Ekedahl. An effective version of Hilbert's irreducibility theorem. In Séminaire de Théorie des Nombres, Paris 1988-1989, Progr. Math. 91, pages 241-249. Birkhaüser Boston, Boston, 1990.

[4] Hooke, R. Linear p-adic groups and their Lie algebras. Ann. of Math. (2) 43, (1942). 641-655.

[5] Looijenga, E. and Peters, C. Torelli theorems for Kähler K3 surfaces. Compositio Math. 42 $(1980 / 81)$, no. 2, 145-186.

[6] Serre, J.-P. Abelian $l$-adic representations and elliptic curves. Second edition. Advanced Book Classics. Addison-Wesley Publishing Company, Advanced Book Program, Redwood City, CA, 1989 